\title{
THE USE OF DENATURED GELATIN FOR BIOCHEMICAL TESTS BY
}

\author{
J. KOHN \\ From Queen Mary's Hospital, Roehampton, London
}

(RECEIVED FOR PUBLICATION MARCH 30, 1953)

This is a preliminary report on the use of denatured gelatin for biochemical tests by saturating it with solutions of substances to be tested.

\section{Sugar Fermentation Tests}

The media are prepared from Coignet's gold medal gelatin in a manner similar to that described for the liquefaction test. Instead of sheet gelatin, Difco nutrient gelatin in about double strength poured out to form sheets can also be used with equally satisfactory results. After formolization the gelatin sheets are cut or punched into shapes, about $15 \mathrm{~mm}$. diameter, convenient for identification. The gelatin pieces are then wrapped in gauze and placed under running water for 24 hours. They are now put into screw-capped containers, and $15 \%$ sugar solutions in water are poured into the containers so as to cover the gelatin pieces. The bottles with their contents are then steam sterilized for $\mathbf{3 0}$ minutes or tyndallized. The sugar solutions are left in contact with the gelatin for about 24 hours and are then decanted. The saturated gelatin pieces are then ready for use. For sugars which are not readily soluble at room temperature, the solutions should be heated before decanting in order to dissolve the sugar. Glucose and lactose require a slightly different procedure, as they tend to undergo some breakdown when sterilized together with the gelatin. For these sugars the gelatin is sterilized separately and sterile solutions of these sugars are added later. The remainder of the procedure is the same. The basic medium for the fermentation tests is bromothymol blue agar, containing 3\% of a $0.5 \%$ alcoholic solution of bromothymol blue. The $p \mathrm{H}$ of the medium should be approximately 7.2.

The organisms to be tested are streaked on to the agar, and the sugar gelatins are placed on the surface of the inoculated agar at suitable intervals. Cross diffusion can be prevented by cutting the agar and judiciously spacing the gelatins. It is advisable to inoculate heavily those spots where the gelatin with be placed. Incubation is at $37^{\circ} \mathrm{C}$.

Alternatively, bromothymol blue solution can bi added to the sugar solutions before sterilization, the gelatin becoming saturated with the sugar and thè indicator at the same time. In this case ordinarf nutrient agar is used as a medium.

Acid production is revealed by the change in coloui of the gelatin and of the underlying medium, gaફ5 showing up as bubbles under the transparent gelatinen After some time the gas tends to escape, but its pres:ence is revealed by rings of precipitate in places where it has been previously produced. Gelatin liquefaction does not interfere with sugar fermentation tests, ag the acid produced by sugar fermentation is sufficien? to inhibit the action of the gelatinases.

Other biochemical tests as, for example, $\mathrm{H}_{2} \mathrm{~S}$ pro duction may be tested in a similar way by saturating the gelatin with a bismuth or iron salt. The use ob denatured gelatin for other tests is under trial.

\section{To the Editor, The Journal of Clinical Pathology}

SIR,-I regret to have to point out that an erroth has crept into my article "A Simple Apparatus fop the Estimation of the Diffusible Fraction of Calciums in Serum" (J. clin. Path., 6, 155) in the sentencefollowing the specimen calculations on p. 156, which should read, "If high accuracy is not required, the result correct to $\pm 1 \mathrm{mg}$. may be read off from Fig. 2."음

Two nomograms, of the accuracy originally visual ized, which have been privately printed on the dis? covery of the mathematical error involved in com pressing and combining them for publication, can be obtained from the author by anyone interested.

I much regret that this should have arisen and that the discrepancy due to compression was not madew clear in the original paper.-Yours, etc.,

G. I. M. Carlier 